\title{
Bridge over troubled water: milk fat globule epidermal growth factor 8 promotes human monocyte-derived macrophage clearance of non-blebbing phosphatidylserine-positive target cells
}

\author{
Cell Death and Differentiation (2007) 14, 1063-1065. doi:10.1038/sj.cdd.4402096; published online 26 January 2007
}

Dear Editor,

Externalization of phosphatidylserine (PS) serves as a recognition signal for neighboring phagocytes, ${ }^{1}$ and studies published 10 years ago demonstrated that blebs on the surface of apoptotic cells are sites of enhanced procoagulant activity, perhaps owing to the aggregation of PS molecules on these membrane protrusions. ${ }^{2}$ However, the putative importance of plasma membrane blebbing for engulfment of dying cells has not been investigated. Phagocytes may not only recognize PS directly, but also secrete so-called opsonins or bridging molecules that bind PS on the dying cell, thereby facilitating corpse clearance. Indeed, deletion in mice of milk fat globule epidermal growth factor 8 (MFG-E8), a PS-binding bridging molecule, ${ }^{3}$ results in impaired engulfment of apoptotic cells and autoimmune disease, thus demonstrating the physiological importance of PS-mediated cell clearance. ${ }^{4}$ Moreover, the involution of mammary glands is impaired in the absence of MFG-E8, indicating that this bridging molecule also plays a role in the removal of apoptotic cells during organ remodelling. ${ }^{5}$ In the present study, we show that plasma membrane blebbing and PS externalization can be dissociated during apoptosis and, moreover, that non-blebbing target cells are less efficiently ingested when compared with their blebbing counterparts. MFG-E8 was found to restore clearance of non-blebbing target cells by human macrophages.

Fas stimulation of Jurkat T cells induced vigorous blebbing of the plasma membrane and activation of caspase-3-like enzymes (Figure 1a and b). These cells also expressed PS on the cell surface, as demonstrated by labelling with fluorescein isothiocyanate (FITC)-conjugated annexin $\mathrm{V}$ (Figure 1c). Preincubation of Jurkat cells with cytochalasin B (CB), a fungal metabolite that prevents actin polymerization and promotes the disruption of the microfilament network, completely abrogated blebbing, yet failed to diminish other features of the apoptotic program. Similar results were obtained when Y-27632, a specific inhibitor of ROCK I kinase, ${ }^{6}$ was tested (Supplementary information, Figure S1). In contrast, the pan-caspase inhibitor, zVAD-fmk, prevented all of the aforementioned apoptotic events. To address whether plasma membrane blebbing is reversible, CB was added to the medium after $3 \mathrm{~h}$ of treatment with agonistic antiFas antibodies, at which time marked blebbing was seen. Jurkat blebbing was completely reversed under these conditions, whereas poly(ADP-ribose) polymerase cleavage and
PS exposure remained unaffected (Supplementary information, Figure S2). In addition, when Jurkat cells were triggered to undergo apoptosis by staurosporine (STS), an inhibitor of protein kinases, no blebbing was seen despite a considerable degree of PS exposure (Figure 1d), consistent with the notion that apoptotic membrane blebbing is controlled by phosphorylation. ${ }^{7}$ Blebbing, thus, appears to be a dynamic event that can be divorced from other features of apoptosis even under conditions when cells are committed to die.

Next, we aimed to test the importance of plasma membrane blebbing for phagocytosis. Fas-stimulated Jurkat cells were readily ingested upon cocultivation with the murine J774A.1 macrophage cell line (Supplementary information, Figure S3); however, the degree of phagocytosis was significantly reduced when Jurkat cells were pretreated with $\mathrm{CB}$. Comparable data were obtained when apoptotic Jurkat cells pretreated or not with CB were added to the human THP.1 macrophage cell line (Supplementary Figure S3). Extensive washing of Jurkat cells was performed before cocultivation in order to exclude a direct effect of residual amounts of $C B$ on macrophages. Pretreatment of Fas-triggered Jurkat cells with Y-27632, an inhibitor of membrane blebbing, also suppressed phagocytosis (Supplementary Figure S1). Moreover, STStriggered (non-blebbing) Jurkat cells were less efficiently engulfed by THP.1 macrophages when compared to Fastriggered (blebbing) target cells (Supplementary Figure S3). These results indicate that PS externalization in the absence of blebbing may be insufficient to render dying cells appetizing to phagocytes. One explanation for this conundrum is that the pattern of PS distribution on the surface of apoptotic cells may determine whether PS is recognized by its corresponding phagocytic receptor(s). CED-1, a phagocytosis receptor in Caenorhabditis elegans, was previously shown to cluster around dying cells, and it was concluded that this receptor clustering was a direct response to a recognition signal, possibly PS, that distinguishes cell corpses from living cells. ${ }^{8}$ Furthermore, studies of galectin-1-treated human T-cell lines have shown that PS localizes to apoptotic blebs protruding from the cell surface. ${ }^{9}$ In line with these observations, our confocal microscopic analysis of Fas-triggered Jurkat cells are suggestive of a colocalization of annexin $V$ with cholesterol-rich lipid raft domains in the plasma membrane (Supplementary information, Figure S4). In sum, although we cannot exclude the possibility that CB and Y-27632 inhibit 

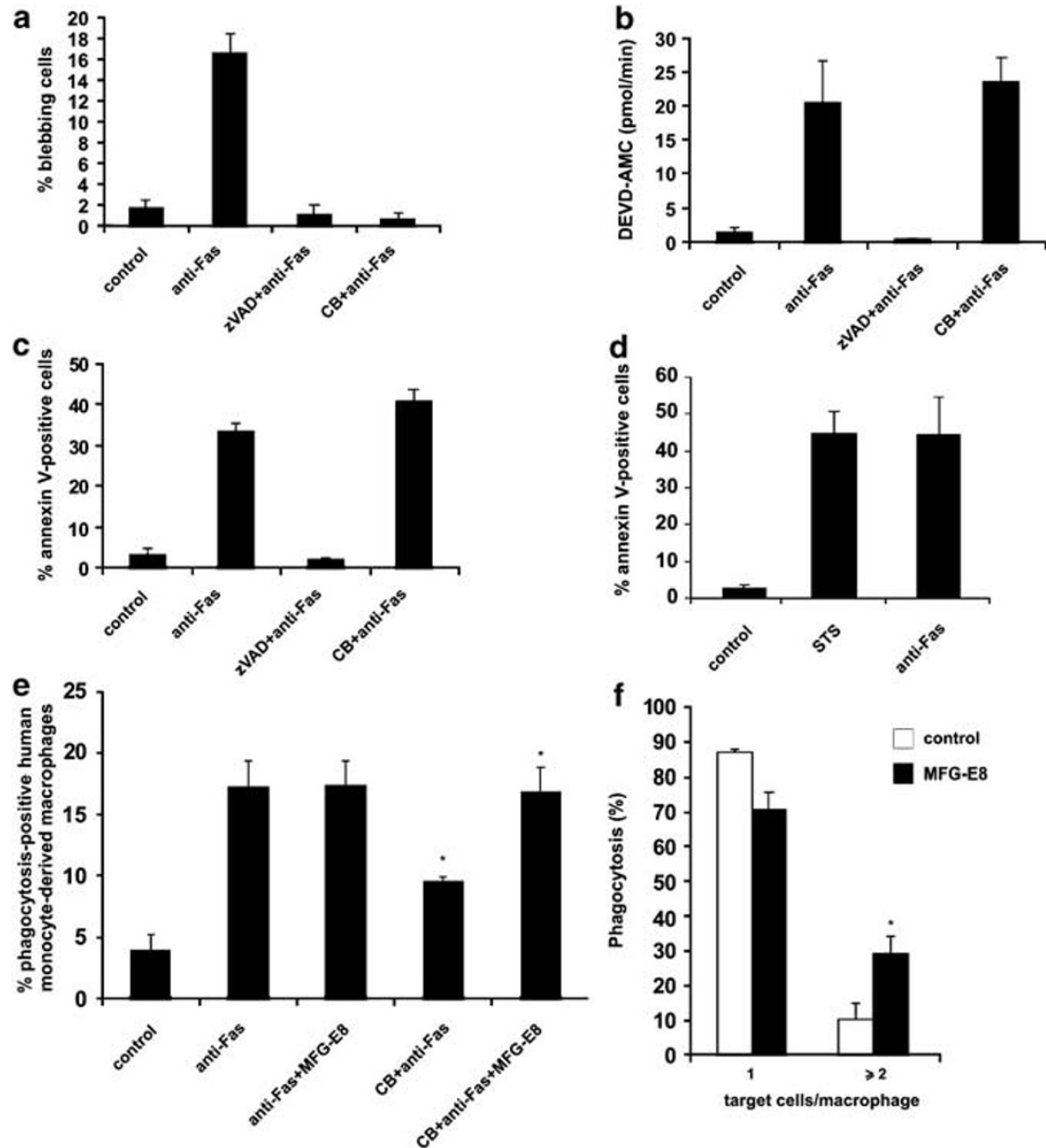

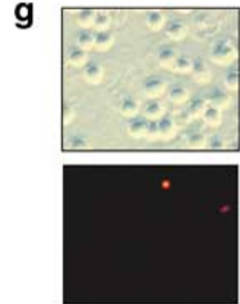

control
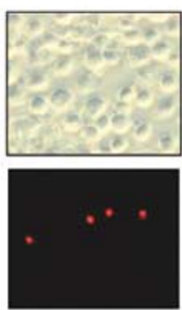

anti-Fas
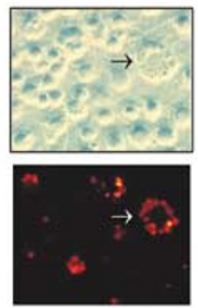

anti-Fas+MFG-E8

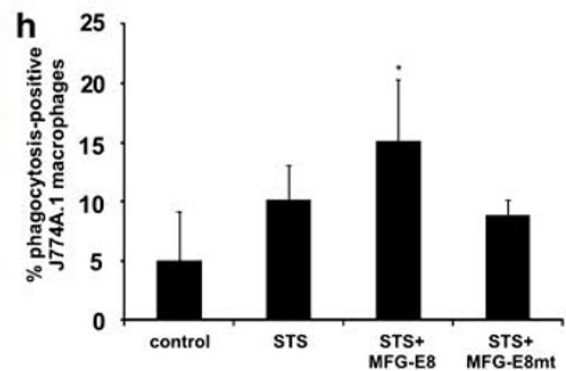

Figure 1 MFG-E8 restores engulfment of non-blebbing target cells. (a) Jurkat cells were incubated in the presence or absence of the pan-caspase inhibitor, zVAD-fmk $(10 \mu \mathrm{M}$, Enzyme Systems Products, Livermore, CA, USA) or the cytoskeleton-disrupting agent, CB ( $5 \mu \mathrm{g} / \mathrm{ml}$, Sigma, St. Louis, MO, USA) for $30 \mathrm{~min}$ and then treated or not with agonistic anti-Fas antibodies ( $250 \mathrm{ng} / \mathrm{ml}$, Medical \& Biological Laboratories, Nagoya, Japan) for $4 \mathrm{~h}$. Blebbing cells were counted under the light microscope. (b) Jurkat cells were stimulated as above and cell lysates were mixed with the fluorogenic substrate, DEVD-AMC ( $50 \mu \mathrm{M}$, Peptide Institute, Osaka, Japan). The rate of AMC release, indicative of caspase-3 activation, was then determined as described. ${ }^{1}$ Data shown in (a and b) are mean values \pm S.D. ( $n=3$ ). (c) Flow cytometric detection of PS exposure in Jurkat cells treated as above using FITC-conjugated annexin V (Oncogene Research Products, Cambridge, MA, USA). Cells were analyzed on a FACScan operating with CellQuest software (BD Biosciences, San Jose, CA, USA). The percentages of annexin V-positive and propidium iodide-negative cells are depicted. Data shown are mean values \pm S.D. $(n=4)$. (d) Quantification of PS exposure in Jurkat cells stimulated with STS $(1 \mu \mathrm{M}$, Sigma) for $2 \mathrm{~h}$ or anti-Fas antibodies $(250 \mathrm{ng} / \mathrm{ml})$ for $4 \mathrm{~h}$ was performed as described above. Data are depicted as mean values \pm S.D. $(n=5)$. The percentage of blebbing cells in the STS-treated samples was negligible $(0.4 \pm 0.3)$. (e) Jurkat cells pretreated or not with $\mathrm{CB}(5 \mu \mathrm{g} / \mathrm{ml})$ were incubated in the presence or absence of Fas-antibodies $(250 \mathrm{ng} / \mathrm{ml})$ for $4 \mathrm{~h}$ and cocultured with HMDMs. Recombinant murine MFG-E8 $(1 \mu \mathrm{g} / \mathrm{ml})$ was added to the co-cultures and the percentages of phagocytosis-positive macrophages were determined as described. ${ }^{1}$ ( $f$ ) Co-cultivation of apoptotic Jurkat cells and HMDMs was performed as in (e) and the percentages of macrophages that carried 1 or $\geqslant 2$ target cells in the presence or absence of MFG-E8 $(1 \mu \mathrm{g} / \mathrm{ml})$ were determined. Data shown in (e and f) are mean values \pm S.D. $(n=3)$. ${ }^{*} P<0.01$. (g) Representative images depicting HMDM engulfment of TAMRA-labelled target cells in the presence or absence of MFG-E8 $(1 \mu \mathrm{g} / \mathrm{ml})$. Jurkat cells were preincubated with CB $(5 \mu \mathrm{g} / \mathrm{ml})$ before Fas stimulation. Phase contrast and fluorescence images were captured using a Nikon Eclipse E600 microscope equipped with a DS-5M digital camera operating with NIS elements software. Arrows indicate a 'super-efficient' macrophage that has ingested numerous apoptotic target cells. Original magnification $\times 40$. (h) Jurkat cells treated with STS $(1 \mu \mathrm{M})$ for $2 \mathrm{~h}$ were cocultured with J774A.1 macrophages (known to use the integrin-receptor system for recognition of apoptotic cells) ${ }^{15}$ in the presence or absence of MFG-E8 or the D89E mutant and the percentages of phagocytosis-positive macrophages were determined as above. Data are reported as mean values \pm S.D. $(n=3) .{ }^{*} P=0.02$ 
other events besides blebbing that contribute to efficient phagocytosis, our combined data nevertheless suggest that the clustering of PS on membrane protrusions or blebs may play a role in corpse clearance.

MFG-E8 is a PS-binding molecule that is secreted by certain subsets of macrophages and dendritic cells. ${ }^{3}$ We reasoned that MFG-E8 should bind to PS molecules on the surface of apoptotic cells irrespective of whether or not these cells are blebbing, and thus serve to promote phagocytosis. Indeed, recombinant FLAG-tagged MFG-E8 was found to bind Fas-stimulated Jurkat cells, but not to untreated cells (data not shown). The degree of MFG-E8 binding to apoptotic Jurkat cells was similar in the presence or absence of CB and correlated with the percentage of PS-positive cells. MFG-E8 was then added to cocultures of Jurkat cells and human monocyte-derived macrophages (HMDMs) that express the $\alpha_{v} \beta_{3}$ integrin receptor to which MFG-E8 binds, ${ }^{10}$ and the degree of phagocytosis was determined. HMDM-mediated clearance of non-blebbing Jurkat cells was diminished when compared with blebbing Jurkat cells (Figure 1e). MFG-E8 restored phagocytosis of non-blebbing cells, whereas no increase in the percentage of phagocytosis-positive macrophages was seen when MFG-E8 was added to blebbing cells. Previous studies in a murine model system have indicated that MFG-E8 may increase the phagocytic index, that is, the number of apoptotic target cells engulfed per macrophage. ${ }^{10}$ Careful enumeration of apoptotic Jurkat cells contained within each macrophage revealed a significant increase in the phagocytic index upon addition of MFG-E8 (Figure 1f) Indeed, numerous instances of highly efficient HMDMs (i.e., macrophages that had ingested $\geqslant 8$ target cells) were observed in MFG-E8-treated cultures (Figure 1g), whereas such cells were never seen in the absence of MFG-E8. Finally, recombinant MFG-E8 also promoted macrophage engulfment of STS-treated Jurkat cells (Figure 1h), thus supporting the notion that uptake of PS-positive, non-blebbing cells is enhanced by this bridging protein. The recombinant D89E mutant form of MFG-E8, ${ }^{10}$ however, did not affect macrophage uptake of target cells.

In conclusion, the present study demonstrates that plasma membrane blebbing can be dissociated from other features of the apoptotic program, including the exposure of PS. Furthermore, our data suggest that blebbing may play a role in the recognition and clearance of apoptotic cells by macrophages, insofar as these membrane protrusions may provide a context for the aggregation of PS molecules. Our results are thus congruent with recent studies implicating PS clustering on the cell surface in the selective engulfment of apoptotic versus non-apoptotic cells. ${ }^{11}$ The current observation that the
PS-binding protein MFG-E8 can restore clearance of nonblebbing target cells by human macrophages could be explained either by the potential ability of this protein to cluster PS molecules on apoptotic cell membranes or by direct effects of MFG-E8 on phagocytes. ${ }^{12}$ Finally, although deletion of MFG-E8 in mice results in autoimmune disease, ${ }^{13}$ silencing of the expression of several $C$. elegans genes containing motifs similar to those of MFG-E8 fails to produce any significant phenotype (D Xue, personal communication). Hence, although PS-dependent mechanisms of corpse clearance have been conserved through evolution, ${ }^{14}$ the involvement of bridging molecules in this process may be specific to mice and men.

Acknowledgements. We thank Bertrand Joseph and Ulrika Nyman for expert advice on confocal imaging and Susanne Virding for assistance in the production of recombinant MFG-E8 protein. This study was supported by grants from the Swedish Research Council, the Swedish Medical Society, the Jeansson Foundation and the Human Frontier Science Program.

\section{E Witasp ${ }^{1,6}$, W Uthaisang ${ }^{2,6}$, C Elenström-Magnusson ${ }^{3}$, R Hanayama ${ }^{4}, M$ Tanaka $^{4}, S_{\text {Nagata }}{ }^{4}$, S Orrenius $^{5}$ and $B$ Fadeel F, $^{*}$ \\ ${ }^{1}$ Division of Biochemical Toxicology, Institute of Environmental Medicine, Karolinska Institutet, Stockholm, Sweden; \\ 2 Department of Biochemistry, Faculty of Science, Mahidol University, Bangkok, Thailand; \\ ${ }^{3}$ Pharmacia Diagnostics AB, Uppsala, Sweden; \\ 4 Department of Genetics, Osaka University Medical School, Osaka, Japan and \\ ${ }^{5}$ Division of Toxicology, Institute of Environmental Medicine, Karolinska Institutet, Stockholm, Sweden \\ 6 These two authors contributed equally to this work \\ * Corresponding author: B Fadeel, Cell Death Research Group, Division of Biochemical Toxicology, Institute of Environmental Medicine, Karolinska Institutet, Nobels väg 13, Stockholm 17177 , Sweden. \\ Tel: + 468524877 37; Fax: + 4683438 49; \\ E-mail: bengt.fadeel@ki.se}

\footnotetext{
1. Kagan VE et al. J Immunol 2002; 169: 487-499.

2. Casciola-Rosen L et al. Proc Natl Acad Sci USA 1996; 93: 1624-1629.

3. Borisenko GG et al. Cell Death Differ 2004; 11: 943-945.

4. Hanayama R et al. Science 2004; 304: 1147-1150.

5. Hanayama R, Nagata S. Proc Natl Acad Sci USA 2005; 102: 16886-16891.

6. Sebbagh M et al. Nat Cell Biol 2001; 3: 346-352.

7. Mills JC et al. J Cell Biol 1998; 140: 627-636.

8. Zhou Z, Hartweig E, Horvitz HR. Cell 2001; 104: 43-56.

9. Pace KE et al. J Immunol 1999; 163: 3801-3811.

10. Hanayama R et al. Nature 2002; 417: 182-187.

11. Appelt U et al. Cell Death Differ 2005; 12: 194-196.

12. Akakura $S$ et al. Exp Cell Res 2004; 292: 403-416.

13. Hanayama R et al. Curr Dir Autoimmun 2006; 9: 162-172.

14. Fadeel B, Xue D. Cell Death Differ 2006; 13: 360-362.

15. Pradhan D et al. Mol Biol Cell 1997: 8:767-778.
} 